\title{
Evaluation of the effectiveness of arthroscopic rotator cuff repair at the Thong Nhat Hospital in Vietnam
}

\author{
V.T. Toan and N.M. Duong \\ Department of Orthopedic and Trauma Surgery, Thong Nhat Hospital, Ho \\ Chi Minh city, Vietnam \\ Corresponding author: V.T. Toan \\ E-mail: vothantoan1990@yahoo.com
}

Genet. Mol. Res. 20 (1): gmr18745

Received December 30, 2019

Accepted April 20, 2020

Final Revision January 25, 2021

Published March 30, 2021

DOI http://dx.doi.org/10.4238/gmr18745

\begin{abstract}
We evaluated the results of arthroscopic rotator cuff repair. Thirty cases of rotator cuff tear were treated by arthroscopic rotator cuff repair at the Thong Nhat hospital from 1/3/2012 to $30 / 6 / 2016$. All patients were over 18 years old, had positive rotator cuff tear tests, and underwent X-ray and magnetic resonance imaging. The arthroscopic images showed rotator cuff tears, with indication for repair. The functional status of each patient was evaluated using the Constant-Murley Scale and the criteria of the University of California at Los Angeles (UCLA). During postoperative rehabilitation, we applied Cohen's protocol. Seventeen patients were female and 13 were male. The median age was 54 years (21-83). Sixteen patients had partial thickness tears and 14 patients had full-thickness tears. Repair technique: single-row in 13 patients, suture-bridge in 15 patients, transosseous-equivalent in two patients. Four patients with SLAP lesions were treated by debridement labrum for type I, and labral repair for more serious lesions. Five patients with long head biceps tendon rupture were treated with tenodesis. After surgery, $100 \%$ of patients were subjected to fluid extravasation through the shoulder joint, but did not have any compartment syndrome; all incisions healed good without infection or neurapraxia. The mean UCLA score was 32.4 (14 patients had excellent results, 14
\end{abstract}


good, and two fair). The mean Constant score was 38.5; the mean postoperative Constant score was $88.77(\mathrm{p}=0.001)$. Among the patients, $90 \%$ achieved $80 \%$ function compared to a normal shoulder. All patients gave their consent to the study and were prepared for the full course of treatment and arthroscopic surgery. Repair of the rotator cuff was for some participants the only effective treatment and a way to restore the shoulder. Postoperative reconstruction of the rotator cuff of the shoulder resulted in a significant improvement in the indicators of the functional state of the shoulder joint.

Key words: Rotator cuff tear; Shoulder arthroscopy

\section{INTRODUCTION}

Rotator cuff tear is a common cause of pain and disability among adults. $10 \%$ to $40 \%$ of people over 40 years old had rotator cuff problems (Coudane and Goutallier, 1997). A torn rotator cuff will weaken and reduce the shoulder functions. Therefore, many daily activities may become painful and difficult to do.

Operative treatment with rotator cuff suture was described by Codman (1911). That method brought about $60-70 \%$ effectiveness in rehabilitation but it had deltoid muscle hypertrophy complications. In recent years, arthroscopic rotator cuff repair has shown more advantages in the evaluation of rotator cuff injuries and repair. This arthroscopic technique has been shown to improve clinical and functional outcomes by over $90 \%$.

In Vietnam, early diagnosis and rotator cuff repair had been focused on since 2000. From 2012, arthroscopic rotator cuff repair was conducted at Thong Nhat Hospital. Therefore, this study aimed to evaluate the effectiveness of arthroscopic rotator cuff repair.

From the point of view of philosophy, due to which the rotator cuff surgery came to the arthroscopic method, the breakthrough was in the desires to increase the ability to visualize to diagnose injuries, minimize tissue damage during medical procedures, correctly recognize pathologies, and optimize reparative work (Buerba et al., 2016; Elhassan et al., 2016). Thanks to arthroscopy, surgeons were able to diagnose problems and perform treatment without prolonged observation and tissue damage, which no longer entailed a long recovery (Deranlot et al., 2017). However, special exercises still have to be done (Saul et al., 2016).

The above goals and objectives for improving the indicators of the functional state of the joints have become fundamental in the creation and development of new surgical approaches and methods. Over the past 25 years, the quality of medicine has been significantly improved; arthroscopic treatment was invented, and this has greatly benefited patients and surgeons. Arthroscopic repair of the rotator cuff in the United States has become increasingly widespread and has become the focus of numerous recent studies (Day et al, 2019). Some studies have also shown that combined surgery with arthroscopic support gives better results when combined with partial cuff repair than when performed in isolation for a massive, irreparable posterior superior rotator cuff tear (Valenti et al., 2017). The apparatus is still being improved to avoid any inflammation and discomfort during and after operations (Paribelli et al., 2015). 


\section{MATERIAL AND METHODS}

\section{Participants}

Participants of the study were selected according to the following inclusion and exclusion criteria.

Inclusion criteria. Patients over 18 years old with:

Clinical examination with positive tests of rotator cuff tear.

X-ray: sclerosis or osteolysis of humeral greater tuberosity, decreased acromiohumeral interval, humeral subluxation superiorly, and/or MRI with partial or complete rotator cuff tear.

Arthroscopic diagnosis: patients had rotator cuff tear and indication for repair.

No improvement after 12 weeks of conservative treatments. Exclusion criteria. Patients did not meet these conditions.

All patients signed a voluntary informed consent to participate in the study. The study design, informed consent form was reviewed and approved by the institutional bioethics experiment and the expert ethics committee.

\section{Methods}

From March 1, 2012 to June 30, 2016, 30 patients were enrolled. Participants were followed by using medical record during this study. The affected shoulder function was evaluated using the Constant score (Constant Shoulder Outcome, 2020).

During surgery: endotracheal anesthesia, the patient was placed in the lateral decubitus position using a bean bag type support placed on the torso and pelvis on a standard operating table, the arm was pulled through the frame using 3-5 kilograms objects. Through one puncture, 1-2 centimeters long, a video camera (arthroscope) is inserted into the joint and all injuries are examined from the inside. Through one to two other small punctures, special instruments are inserted into the joint, with which the tendons are sutured. (Ghodadra et al., 2009) .

Portal placement: posterior, anterior, lateral, posterolateral, and auxiliary portal placements. Evaluating of the rotator cuff tear and concomitant injuries. Glenoid labrum tear was sutured in type II and underwent debridement of the type

I. Biceps tendon tenodesis was used if it was a nearly complete tear.

Rotator cuff suture used single-row or double-row repair techniques. A postoperative rehabilitation protocol was conducted using the Cohen program (Cohen et al., 2002). The affected shoulder of the patients was evaluated using the Constant score and the UCLA score (UCLA Shoulder Score, 2020). MRI was used for tendon healing assessment. Data were analyzed using Statistical Package for Social Sciences (SPSS) Program, version 20.0 .

\section{Baseline characteristics}

Among 30 patients, there were 17 females and 13 males. Median age was 54 (2183). Median age of the female group was 54 (21-76) and for the male group it was 52.6 (34- 
83). Among 30 patients with rotator cuff tear, there were 23 participants aged from 45 to 65 (76.7\%).

Table 1. Distribution of patients who underwent arthroscopic rotator cuff repair by age group.

\begin{tabular}{ll}
\hline Age group & Number of patients \\
\hline$<35$ & 1 \\
$35 \leq<45$ & 3 \\
$45 \leq<55$ & 14 \\
$55 \leq<65$ & 9 \\
$\geq 65$ & 3 \\
\hline
\end{tabular}

The median follow-up time was 29 months (11-51).

To study the effectiveness of the arthroscopy in case of rupture or damage of the cuff, patients with injuries that could not be repaired except by surgical intervention were selected. The treatment of the shoulder cuff consists in re- placing the torn tendon in its anatomical place to restore all its functions. This is done using arthroscopy, that is, without opening the shoulder joint. Access is through small openings smaller than $5 \mathrm{~mm}$. Thus, arthroscopy does not violate the anatomical structures (Kluger et al., 2011; Valenti et al., 2017).

\section{RESULTS}

\section{Rotator cuff tear}

There were 16 patients with rotator cuff partial thickness tear and 14 patients with full-thickness tear. In the group with rotator cuff full- thickness tear, there was pme case with mild tear, nine moderate tear, three large tear, and one massive tear.

\section{Rotator cuff repair}

Insensitive method. All participants were undergoing endotracheal anesthesia, and used bupivacaine in postoperative pain relief.

Tendon suture methods. Among 30 patients, there were 13 patients using single-row repair, 15 patients with suture-bridge technique, and two patients using transtendon-repair technique.

Concomitant injuries included four patients with type I SLAP tear which required debridement, and suture for other types. Five patients with biceps tendon tear were treated by long head of biceps tendon tenodesis.

\section{Treatment results}

Postoperative complications. All patients had fluid extravasation but no compartment syndrome complications and nerve injuries. No patient had infected surgical incisions. 


\section{Treatment outcome}

Pre-and postoperative Constant score. Median preoperative Constant score was 38.5 compared with 87.77 postoperative. There was a significant difference between pre- and postoperative Constant score $(\mathrm{P}=0.001)$.

Postoperative UCLA score. Median postoperative UCLA score was 32.4. The results were 14 excellent (46.67\%), $14 \operatorname{good}(46.67 \%), 2$ fair $(6.67 \%)$, no patient with poor result. In group of patients aged over 65, there were 1 excellent and 1 good result.

In this study, patients were evaluated and compared postoperative shoulders function - affected shoulder and normal shoulder by using the Constant score. 20 patients were enrolled. After surgery, $75 \%$ patients with affected shoulder achieve $90 \%$ of function compared with normal shoulder. In addition to, $90 \%$ patients recovered $80 \%$ of function compared to normal shoulder.

Postoperative shoulder function of rotator cuff full-thickness tear group and partial thickness tear group. A number of 11 patients with rotator cuff partial thickness tear had postoperative median Constant score 87.95. 13 patients with full-thickness tear had score 87.57. There was no significant difference between two groups $(\mathrm{P}=0.25)$.

Postoperative median Constant score of female and male groups. Postoperative median Constant score of female group was 87.85 compared with male group 87.65 and there was no significant difference between the two groups $(\mathrm{P}=0.9)$. Postoperative UCLA score of rotator cuff full-thickness tear group and partial thickness tear group. There was no significant difference between rotator cuff full-thickness tear group and partial thickness tear group $(P=0.7)$. There was no significant difference in the UCLA score between male and female groups $(\mathrm{P}=0.82)$.

Correlation between age and postoperative Constant score gave a regression equation (postoperative Constant score) $=-0.19$ (age) +98.05 . Therefore, there was a negative correlation between postoperative Constant score and age. There was no significant difference in linear correlation between postoperative UCLA score and age ( $\mathrm{P}=$ $0.28)$.

There was no significant difference in linear correlation between operative time and postoperative Constant score $(\mathrm{P}=0.074)$. Therefore, postoperative results were not affected by operative time. There was no significant difference in linear correlation between operative time and postoperative UCLA score $(\mathrm{P}=0.337)$.

In this study, there were nine patients with concomitant injuries. Five patients had partial or complete long head of biceps tendon tear and four patients had glenoid labrum tear. The other 21 patients had no concomitant injuries.

Preoperative Constant score of rotator cuff tear combined SLAP injuries group and only rotator cuff tear group comparison showed no significant difference in preoperative Constant score between the two groups $(\mathrm{P}=0.49)$.

Postoperative UCLA score of rotator cuff tear combined SLAP injuries group and only rotator cuff tear group comparison showed no significant difference in postoperative UCLA score between two groups $(\mathrm{P}=0.3)$. Postoperative Constant score of rotator cuff tear combined SLAP injuries group and only rotator cuff tear group comparison showed no significant difference in postoperative Constant score between the two groups $(\mathrm{P}=0.23)$. Preoprative Constant score of rotator cuff tear combined long head of biceps tendon tear 
group and only rotator cuff tear group showed no significant difference in preoperative Constant score between the two groups $(\mathrm{P}=0.81)$.

\section{Postoperative shoulder function}

Table 2. Distribution of patients who underwent arthroscopic rotator cuff repair by follow-up time.

\begin{tabular}{lllc}
\hline Follow-up time & Under 12 months & $\mathbf{1 2 - 2 4}$ months & Over 24 months \\
\hline Number of patients & 2 & 8 & 20 \\
\hline
\end{tabular}

Table 3. Postoperative Median Constant score (Yian and Ramappa, 2015).

\begin{tabular}{llll}
\hline Groups & Median score & Standard deviation & Confidence intervals (95\%) \\
\hline Under 12 & 91.43 & 1.43 & $87.93-94.93$ \\
months & 86.9 & 1.88 & $83.08-90.71$ \\
12-24 months & 87.86 & 0.87 & $86.13-89.59$ \\
Over 24 months & & & \\
\hline
\end{tabular}

The study of Kluger et al. (2011) showed that most repeated rotator cuff tear happened in the first 3 months, 6 months (14\%) and caused by trauma. According to the Galatz et al. (2004) study, patients with large or massive rotator cuff tear had good results in the first year despite high repeated tear rate later. However, after 2 years' follow-up, there was a drop in shoulder function. In this study, there was no significant difference in Constant, UCLA score between groups divided by follow- up time. Thus, there was no shoulder functional reduction in over 24 months' follow-up group compared with other groups.

\section{Complications after arthroscopic rotator cuff repair}

Nerve and blood vessel injuries: Weber, et al. study (2002) showed that nerve injury complications happened in low rate. In this study, there was no nerve and blood vessel injury complications. Thus, arthroscopic rotator cuff repair was a safe surgical technique.

Shoulder stiffness after rotator cuff surgery: According to Burkhart, et al. (2006), the percentage of shoulder stiffness after surgery was $4.9 \%$. In this study, no patient had shoulder stiffness complication. However, $53.33 \%$ of patients suffered from limited shoulder motion, especially internal rotation. Thus, they were recommended for a rehabilitation protocol after surgery.

Fluid extravasation after surgery: Lee H.C, et al. (1992) reported that several patients had subcutaneous emphysema, pneumomediastinum, and potentially lifethreatening tension pneumothorax after arthroscopic rotator cuff repair. In this study, 100\% patients had fluid extravasation but no compartment syndrome complications.

Infection after surgery: Mirzayan et al. (2000) study showed that 13 patients with infected surgical incision after operative rotator cuff suture caused by Staphylococcus epidermidis, Staphylococcus aureus, and Propionibacterium species. Almost all patients had no fever, elevation of white blood cells or VS index but suffered from pain and limited 
shoulder motion. All participants had concomitant diseases such as lymphoma disease, breast cancer, diabetes, hypothyroidism, or Iga deficiency. In this study, no patient had infection after surgery.

Suture anchor pullout after surgery: According to Benson et al. (2010), 6 patients with 9 suture anchor were pull out after surgery. Patients with rotator cuff tear over $3 \mathrm{~cm}$ had rate of suture anchor pullout up to $11 \%$. In this study, no patient had suture anchor pullout after surgery.

Trends show that after proper treatment, the repetition of the cuff rupture is still not minimized, but the rotational cuff itself becomes more elastic, resistant to sprains and other injuries (Murphy et al., 2016). The first year of breaks was less than in subsequent ones. However, the shoulder should be protected from stress for the first six months after surgery, otherwise any injury could result in a rupture (Denard, 2015).

\section{DISCUSSION}

The rotational cuff is a group of muscles and tendons that form the cuff above the shoulder joint. These muscles and tendons hold the hand in the joint and help the shoulder joint to move. Tendons may be torn from excessive stress or injury, but the problem may not be related to sports and may occur in people without an excessive history of work (Hartzler and Burkhart, 2017).

Injuries to the rotator cuff of the shoulder include tendonitis, that is, inflammation and dystrophy of the tendon. Injuries also include complete or partial rupture. Subacromial bursitis may be a consequence of tendonitis. Symptoms are manifested by pain in the shoulder joint, and with severe tears are complemented by weakness. The diagnosis is made immediately by the results of the examination, only sometimes diagnostic studies. Tendinitis usually develops as a result of chronic impingement syndrome of the supraspinatus tendon between the humeral head and the coracoid-acromial arch (acromion, acromioclavicular joint, coracoid process, coracoacromial ligament). Exercises that require repeated lifting of the arms above the head and lifting weights above shoulder level increase the risk of injury to the rotator cuff (Denard and Burkhart, 2016). After this, many other studies were conducted studying the options for restoring the shoulder cuff. Now many methods have been developed by which it is possible to accelerate the restoration of the shoulder after surgery. At the moment, surgery to break the cuff is mandatory. This study shows that arthroscopic restoration of the rotator cuff is one of the safest methods and does not entail a repeated rupture.

A recent study was conducted by the Orthopedic Rizzoli Institute (Veronesi et al., 2020), where two groups of patients with irreversible rupture of the rotator cuff who received surgical treatment were evaluated: one group received the arthroscopic transfer of the tibial tendon (LDTT), and the other received a partial restoration of the arthroscopic rotator cuff. According to the results of the study, it was decided that both methods are effective. Tibial tendon transfer showed significant improvements compared with partial restoration by an arthroscope (Benson et al., 2010). However, arthroscopic restoration proceeds painlessly and with less discomfort, moreover, the restoration phase is more likely to go through. According to studies (Murphy et al., 2016; Sheean et al., 2017), the arthroscopic repair is known to be much safer and less likely to cause complications. Many restorations, such as the transfer of a tendon from another part of the body to the rotator cuff 
(Grimberg et al., 2015), cause inflammation and complications from the position of the nervous system. Recovery is harder and longer. When manipulated by an arthroscope, the opposite is true. Patients operated on with an isolated upper or posterior-upper rotator cuff immobilized with a brace at $15^{\circ}$ ER felt less pain and a better passive range of motion shortly after surgery. In comparison, it is known that arthroscopy of the shoulder joint presents an increased risk of complications compared to arthroscopy of the knee in relation to vascular and neurological injuries, excessive fluid secretion, rigidity, iatrogenic tendon damage, and equipment failure. But with the right approach, these risks can be minimized (Sheean et al., 2017).

The study of the rehabilitation period (Bond et al., 2018) after cuff surgery showed that rehabilitation can be significantly accelerated thanks to a special exercise technique. Patients who receive an early exercise program combined with treatment can restore shoulder function faster and reduce pain and range of motion than those who receive a standard exercise program. To this end, studies are still ongoing, if this is confirmed, the study can be used clinically to improve the recovery of patients with arthroscopic recovery.

Also, in 2017, a local study (Jensen et al., 2017) was conducted by American scientists to assess trends in the open and arthroscopic restoration of the rotator cuff in the United States. It was decided to divide people into groups by gender, age, and region of residence. Prior to the study, there was a growing trend in the popularity of cuff repair in an arthroscopic manner, and the researchers confirmed this observation. As a result, it turned out that the request for repair of the rotator cuff increased by $188 \%$ of the total volume in the last couple of years before the study. Most of the applicants preferred treatment with an arthroscope. The process also proved that gender is not related to the restoration of the shoulder cuff. Both men and women have the same probability of complication or a good recovery period, this does not depend on gender (Jensen et al., 2017).

Studies on the restoration of the rotator cuff are still ongoing. The topic is becoming more relevant every year in the USA and Europe. For the rest of the countries, there is no reliable statistical information. It is proved that elderly people most often suffer from this ailment. Most operations in the US occur in people over the age of 60 (Jensen et al., 2017).

At the moment, arthroscopic shoulder restoration is the safest and most effective solution. After surgery, you can speed up recovery with special exercises, this will help increase the strength of the shoulder (Denard et al., 2015).

Treatment of the rotational cuff of the shoulder with the arthroscopic method contributes to the complete cure of patients and in the improvement of shoulder functions in the future. In younger and healthier patients, the arthroscopic repair is known to occur faster. This treatment option improves performance and strength.

At the moment, there are many more studies on the restoration of the rotational cuff of the shoulder and arthroscopy in general. Surgeons around the world are interested in preventing the cuff from rupturing and in healing it quickly if it is still damaged. Arthroscopy is now the leader in all respects in the treatment of the rotator cuff, it is painless and effective, in addition, the shoulder joint does not open during the operation and the muscles are not damaged.

\section{CONCLUSIONS}

There was a shoulder functional improvement in Constant score between pre- and post-operative rotator cuff repair. According to UCLA score, the postoperative results for 
the 30 patients were 14 excellent, 14 good, and two fair; no patient had a poor result. Therefore, arthroscopic rotator cuff repair brought about a better outcome for patients.

\section{ACKNOWLEDGMENTS}

The authors would like to thank Thong Nhat Hospital for financial support.

\section{CONFLICTS OF INTEREST}

The authors declare no conflict of interest.

\section{REFERENCES}

Barnes LA, Kim HM, Caldwell JM, Buza J, et al. (2017). Satisfaction, function and repair integrity after arthroscopic versus mini- open rotator cuff repair. Bone Joint J. 99B(2): 245-249.

Benson EC, MacDermid JC, Drosdowech DS and Athwal GS (2010). The Incidence of Early Metallic Suture Anchor Pullout After Arthroscopic Rotator Cuff Repair. Arthroscopy. 26(3): 310-331.

Bond EC, Hunt L, Brick MJ, Leigh WB, et al. (2018). Arthroscopic, open and mini-open approach for rotator cuff repair: no difference in pain or function at 24 months. ANZ J. Surg. 88(1-2): 50-55.

Buerba RA, Arshi A, Lee KM, Levine BD, et al. (2017). MRI-Arthroscopy correlation of the rotator cuff: a case-based review. Sports Med. Arthrosc. 25(4): 164-171.

Burkhart SS, Lo IKY and Brady PC (2006). A cowboy's guide to advanced shoulder arthroscopy. Philadelphia. Lippincott Williams and Wilkins.

Codman EA (1911). Complete rupture of the supraspinatus tendon. Operative treatment with report of two successful cases. Boston Med. Surg. J. 162: 708-110.

Cohen BS, Romeo AA and Bach B (2002). Rehabilitation of the shoulder after rotator-cuff repair. Operat. Tech. Orthop. 12(3): 218-224.

Constant Shoulder Outcome Score (2020). Retrieved from https://www.orthobullets.com/basic-science/9087/outcomemeasure-tools

Coudane H and Goutallier D (1997). Pathologie de la coiffe des rotateurs. In Encycl. Méd. Chir. (Elsevier, ParisFrance), Appareil Locomoteur. 14-350-A-10.

Day MA, Westermann RW, Bedard NA, et al. (2019). Trends Associated with Open Versus Arthroscopic Rotator Cuff Repair. HSS Jrnl. 15: 133-136 https://doi.org/10.1007/s11420-018-9628-2.

Denard PJ and Burkhart SS (2016). A load-sharing rip-stop fixation construct for arthroscopic rotator cuff repair. Arthrosc. Tech. 1(1): e37-e42.

Denard PJ, Lädermann A, Brady PC, Narbona P, et al. (2015). Pseudoparalysis from a massive rotator cuff tear is reliably reversed with an arthroscopic rotator cuff repair in patients without preoperative glenohumeral arthritis. Am. J. Sports Med. 43(10): 2373-2378.

Deranlot J, Herisson O, Nourissat G, Zbili D, et al. (2017) Arthroscopic subacromial spacer implantation in patients with massive irreparable rotator cuff tears: clinical and radiographic results of 39 retrospectives cases. Arthroscopy. 33(9): 1639-1644.

Elhassan BT, Wagner ER and Werthel JD (2016) Outcome of lower trapezius transfer to reconstruct massive irreparable posterior-superior rotator cuff tear. J. Shoulder Elb. Surg. 25(8): 1346-1353.

Galatz LM, Ball CM, Teefey SA, Middleton WD, et al. (2004). The outcome and repair integrity of completely arthroscopically repaired large and massive rotator cuff tears. J. Bone Joint Surg. Am. 86(2): 219-224.

Ghodadra N, Provencher M and Wiek K (2009). Open, Mini-open, and All-Arthroscopic Rotator Cuff Repair Surgery: Indications and Implications for Rehabilitation. Orthop. Sports Phys. Ther. 39(2): 81-89.

Grimberg J, Kany J, Valenti P, Amaravathi R, et al. (2015) Arthroscopic-assisted latissimus dorsi tendon transfer for irreparable posterosuperior cuff tears. Arthroscopy. 31(4): 599-607.

Hartzler RU and Burkhart SS (2017). Superior capsular reconstruction. Orthopedics. 40(5): 271-280.

Jensen AR, Cha PS, Devana SK, Ishmael C, et al. (2017). Evaluation of the trends, concomitant procedures, and complications with open and arthroscopic rotator cuff repairs in the Medicare population. Orthop. J. Sports Med. 5(10): 2325967117731310.

Kluger R, Bock P, Mittlbo M, Krampla W, et al. (2011). Long-term survivorship of rotator cuff repairs using ultrasound and magnetic resonance imaging analysis. Am. J. Sports Med. 39: 2071-2081. 
Lee HC, Dewan N and Crosby L (1992). Subcutaneous emphysema, pneumomediastinum, and potentially lifethreatening tension pneumothorax. Pulmonary complications from arthroscopic shoulder decompression. Chest. 101: 1265-1267.

Mirzayan R, Itamura JM, Vangsness Jr T, Holtom PD, et al. (2000). Management of Chronic Deep Infection Following Rotator Cuff Repair. J. Bone Joint Surg. Am. 82: 1115-1121.

Murphy J, Gray A, Cooper C, Cooper D, et al. (2016). Costs, quality of life and cost-effectiveness of arthroscopic and open repair for rotator cuff tears: an economic evaluation alongside the UKUFF trial. Bone Joint J. 98B(12): 16481655 .

Paribelli G, Boschi S, Randelli P, Compagnoni R, et al. (2015). Clinical outcome of latissimus dorsi tendon transfer and partial cuff repair in irreparable postero-superior rotator cuff tear. Musculoskelet. Surg. 99(2): 127-132.

Saul KR, Hayon S, Smith TL, Tuohy CJ, et al. (2016). Postural dependence of passive tension in the supraspinatus following rotator cuff repair: a simulation analysis. Clin. Biomech. 26: 804-810.

Sheean AJ, Hartzler RU, Denard PJ, Lädermann A, et al. (2017). Preoperative radiographic risk factors for incomplete arthroscopic supraspinatus tendon repair in massive rotator cuff tears. Arthroscopy. 34(4): 1121-1127.

UCLA Shoulder Score (2020). Retrieved from https://www.orthobullets.com/basic- science/9087/outcome-measuretools.

Valenti P, Maqdes A and Werthel JD (2017). Clinical and radiological outcomes after reverse shoulder arthroplasty in patients with failed deltoid or latissimus dorsi transfers. A review of ten cases. Intern. Orthop. 41(10): 2143-2148.

Veronesi F, Borsari V, Contartese D and Xian J (2020). The clinical strategies for tendon repair with biomaterials: A review on rotator cuff and Achilles tendons. J. Biomed. Mater. Res. Part B: Appl. Biomater. 108(5): 1826-1843.

Weber SC, Abrams JS and Nottage WM (2002). Complications Associated With Arthroscopic Shoulder Surgery. Arthroscopy. 18(2, Suppl. 1): 88-95.

Yian EH, Ramappa AJ, Arneberg O and Gerber C (2005). The Constant score in normal shoulders. J Shoulder Elbow Surg. 14(2): 128-33. doi: 10.1016/j.jse.2004.07.003. 